\title{
THE INTRA-OCULAR TENSION
}

It is generally stated that if the intra-ocular pressure departs from - the normal in cases of intra-ocular neoplasm, it does so in an upward direction.

This statement is substantially true in the majority of cases, but there are exceptions.

Marshall states " The general opinion is, that if a tumour is suspected in a case of detached retina, and if the tension is raised, the probability is that a tumour exists, and if the intra-ocular pressure is decreased the case is probably one of simple detachment, but even in normal or diminished tensions growths are found."

I think it would not be far from the truth to say, that it is only in the minority of cases that tonometry is employed when an intraocular neoplasm is diagnosed or suspected, and therefore the majority of the records on this point are statements based on the clinical estimation of the intra-ocular pressure, by means of palpation with the fingers.

There is little doubt that this method is satisfactory in cases of gross departure of the pressure from normal, but it is not sufficiently accurate to discover small variations of tension between the two eyes in an individual case, or the pressure in the same eye at different periods of time.

Many cases of intra-ocular neoplasms are seen for the first time comparatively late in the disease and these offer no difficulty with regard to tension, it being obviously increased to palpation and the globes often exhibiting corneal oedema; other cases, such as shrunken globes and those with extra-ocular spread of the growth, exhibit obvious diminution in tension.

It is well known that the intra-ocular tension may be normal in cases of neoplasm and there is evidence in the literature to show that these growths can exist in eyes in which the intra-ocular tension is diminished.

The earliest reference to diminished tension in intra-ocular sarcoma was made by Lawrence. He says "Mr. Nunneley of Leeds told me he had frequently met with soft globes in the first stage of malignant disease of the eyeball."

The first comprehensive study of intra-ocular tension in these cases was carried out by Marshall, and he says " Diminished tension by no means uncommonly occurs when the ciliary body is involved, but I have been unable to find an undoubted case in which the tension has been reduced when the choroid only is involved."

Leber and Krahnstover reviewed the literature on hypotony and reported thirty-seven cases, but they made no attempt to measure 
the intra-ocular pressure in both eyes, and their cases are largely made up of globes which had shrunken.

Franz reported three cases, comparing both eyes, and concluded that in early cases hypotony was not due to bacterial infection or uveal inflammation.

Dunnington analysed fifty-five cases from the point of view of tension and the following table is taken from his work.

\begin{tabular}{llrrrrc} 
Tension & \multicolumn{4}{c}{ No. of Cases } & \multicolumn{3}{c}{ Percentage } \\
Higher & $\ldots$ & $\ldots$ & 15 & $\ldots$ & $\ldots$ & $27 \cdot 3$ \\
Equal & $\ldots$ & $\ldots$ & 11 & $\ldots$ & $\ldots$ & 20.0 \\
Lower & $\ldots$ & $\ldots$ & 29 & $\ldots$ & $\ldots$ & 52.7
\end{tabular}

He states "In nine of the fifteen cases the disease had progressed to the secondary glaucoma stage, and if these are not included, it was found that the intra-ocular tension was equal to or less than the fellow eye in 86.9 per cent. of the remaining 46 cases." He also states that " in none of these cases phthisis bulbi had been found."

Marshall, in his study of intra-ocular tension in neoplasms within the globe, divided his cases into two groups.

A. Sarcoma of choroid only.

B. Sarcoma of the ciliary region.

He found that of fifty-three in group $A$, tension was increased in 36 , or 67.9 per cent., tension was normal in 16 or 30.18 per cent., and tension was ? diminished in 1 , or 1.88 per cent.

Of twenty-eight cases in group $\mathbf{B}$, he found that tension was increased in 10 or 35.7 per cent., tension was normal in 14 or 50 per cent., and tension was diminished in 4 or 14.28 per cent.

He states "It will be seen that the tension is more often increased in choroidal than in ciliary growths, i.e., 50 per cent. of the ciliary growths showed no increase in tension."

Cause of increased tension in intra-ocular neoplasm.-The mechanism of increased intra-ocular tension in globes affected with intra-ocular growths has been the subject of much discussion, and it would be correct to state that at the present time there is no universally agreed cause for this phenomenon, although many theories and statements have been put forward.

Although it is extremely common to find an increased tension, at one period or another during the natural history of the growth, there are many cases which do not exhibit this phenomenon, although they frequently have growths of the same character, size and position which in other globes give rise to marked increase in intra-ocular pressure.

From this one might assume that the size and position of the growth has little, if anything, to do with the presence of increased 
tension, but it must be remembered that these eyes may have been removed at a period before giving rise to increased tension; in other words, they might quite possibly, if left long enough, cause increased intra-ocular pressure later, such as is so commonly seen in those cases of blind painful eyes which, on enucleation, show a surprisingly large percentage of choroidal sarcoma.

I think that the majority of authorities are agreed that, other things being equal, the condition of the filtration angle of the anterior chamber corresponds very closely with intra-ocular pressure.

Closure of this angle interferes with the normal outflow of aqueous from the eye and thereby causes the tension to rise.

Marshall found that the angle was open and the anterior chamber deep in only one case out of thirty-six in which the intra-ocular tension was increased, the remainder having the angle either closed or restricted. Closure of this angle can be brought about in several ways.

1.' The iris can be pushed forward.

2. Formation of a plasmoid type of intra-ocular fluid.

3. Pigmented or growth cells impeding the angle.

When the growth is small and in an early stage of its development, and there is not a large coincident detachment of the retina, it is unusual to find an increase in tension.

When, however, the growth assumes larger proportions and has probably broken through the membrane of Bruch, the vitreous starts to be compressed and, in turn, presses the iris towards the cornea, thus occluding or restricting the angle.

The formation of a plasmoid type of intra-ocular fluid can be obtained in several ways.

In nearly all cases of increased tension there is a detachment of the retina beneath which there is a highly albuminoid type of fluid.

The cause of the detachment is probably brought about by transudation of this fluid from the choroidal vascular bed, and is often quite a separate detachment to that caused by the growth itself.

Haemorrhages or necrosis within the growth are other causes for the formation of this plasmoid type of fluid.

This fluid finds greater difficulty in getting out of the eye than the normal intra-ocular fluids, and thus tends to increase the tension.

It is stated that growth cells have been observed free in the anterior chamber, and it is said that they themselves can be so numerous as to block up the filtration angle. This phenomenon I have personally never observed, nor have I seen any record of such a happening in the pathological reports of the present series of cases. 
Interference with the venous outflow from the globe is another possible cause of increased pressure, the growth interfering with drainage via the vernae vorticosae.

In this series of cases there does not appear to be any true relationship between the position of the tumour within the globe and increased intra-ocular pressure.

The size of the tumour, however, has some relationship to tension, those cases in which the vitreous chamber was full of growth invariably showed increased intra-ocular tension unless the sclerotic had been perforated by growth.

Small tumours, however, in many cases showed increásed intraocular tension, even tumours so small in themselves as to be incapable of interfering with the vitreous and lens. In these cases there was, however, a large secondary detachment, the tension in all probability being due to bulk of inter-retinal fluid and an increase in the viscosity of the intra-ocular fluids.

It is probable, therefore, that there are several factors involved in the production of increased intra-ocular tension.

The closure of, or the restricted outflow of fluid from the filtration angle of the anterior chamber is the all important cause.

This closure is brought about by the lens and iris being pushed forward by vitreous, which, in turn has been moved by either growth or bulk of inter-retinal fluid.

The restricted outflow from the filtration angle is due to the formation of a plasmoid type of intra-ocular fluid.

In the present series, the intra-ocular tension has in the majority of the cases been taken from the record on the clinical notes.

I have, in three cases only, done tonometric measurements, these three cases exhibiting a normal intra-ocular tension to palpation with the fingers.

Two of these showed tonometric readings the same in either eye. The third, however, showed a diminished tension by $1 \mathrm{~mm}$. Hg Schiötz in the affected eye, but this, I consider, was within the realm of experimental error.

Of the 263 cases, increased tension was found to be present in 113 , or 49.4 per cent., and diminished tension in 8 , or 3.04 per cent. of the cases, in 142 cases the tension was normal.

Of the primary ciliary body growths, of which there were 10 , the tension was not found diminished in one. In four cases it was normal to palpation, and in six it was definitely increased, one case registering $115 \mathrm{~mm}$. $\mathrm{Hg}$ Schiötz.

This is not in agreement with Marshall, who found that in 50 per cent. of the primary ciliary body growths there was no increase in tension. 


\section{BIBLIOGRAPHY}

Marshall, C. D.-Tension in cases of intraocular tumours. Trans. Ophthal. Soc. U.K., Vol. XVI, p. 155.1896.

LAWRENCE, W.-Ophthal. Rev., Vol. 2, p. 378.1866.

LEBER, T. and KRAHNSTOVER-Arch.f. Ophthal., Vol. 45, p. 164.1898.

FRANZ, L. M.-Amer. Jl. Ophthal., Vol. 111, p. 872.1920.

DUNNINGTON, J. H.- Intraocular tension in cases of sarcoma of choroid and ciliary body. Arch. Ophthal., Vol. XX. p. 359. 1938.

Neame, H.-Brit. Jl. Ophthal., Vol. 9, p. 618. 1925.

\section{PATHOLOGY}

In many cases of choroidal sarcoma it is difficult or impossible to determine with certainty the point of origin of the tumour when it has spread over a considerable area of the uvea. This difficulty - arises occasionally even when the whole eye is available for pathological examination, and it will be readily realised that the problem is intensified in those cases where one is left with only a section of the original specimen to study. In the following series (see Table A) I have included only those cases in which their origin is certain enough for classification purposes, and I have excluded those tumours which have spread over such a large portion of the choroid as to make them impossible for classification. Examples of these are those tumours that extend from the optic papilla posteriorly, to the ciliary body anteriorly, and those in which the neoplasm occupies the whole or the greater part of the vitreous chamber.

With regard to the remainder of the uveal tract, two zones are easily separated for classification purposes. These are the ciliary body and iris. The remaining divisions in Table A., i.e., the choroid and ciliary body, the ciliary body and iris, and the choroid and iris, are included because it was found that it was impossible to determine in which area the primary growth originated. The solitary case in the last division is interesting, as I have been unable to find any record in the literature of a choroidal sarcoma and a malignant melanoma of the iris occurring in the same eye. It is possible, of course, that the iris growth may be a local metastasis from the choroidal sarcoma, as neoplastic cells have been observed free in the anterior chamber in some cases of choroidal sarcoma.

For choroidal sarcomata three main divisional areas have been employed. Anterior, denoting that area of the uvea between the ciliary body and equator, equatorial, and posterior (see Table B).

Circum-papillary tumours and tumours occurring in the macular region are included in the posterior group, those occurring in the latter region being of extreme interest when their previous clinical history is studied; and several of these cases are described in detail in the appendix. 